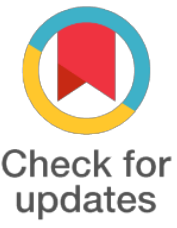

*For correspondence:

Badrul Hisham Yahaya

Competing interests: The authors declare that no competing interests exist.

Received: 2017-04-30

Accepted: 2017-06-10

Published: 2017-09-05

Copyright The Author(s) 2017. This article is published with open access by BioMedPress (BMP).

This article is distributed under the terms of the Creative Commons Attribution License (CC-BY 4.0) which permits any use, distribution, and reproduction in any medium, provided the original author(s) and the source are credited.

\section{Lung cancer stem cell and its therapeutic potential}

\author{
Badrul Hisham Yahaya*, Norashikin Zakaria, Noor Hanis Abu Halim, Nazilah Satar \\ Regenerative Medicine Cluster, Advanced Medical and Dental Institute, Universiti Sains Malaysia, \\ Bertam, 13200 Kepala Batas, Pulau Pinang, Malaysia
}

\section{Abstract}

Cancer stem cells (CSCs), a subpopulation of cancer cells with the ability to self-renew and giving rise to differentiated cancer cells, are thought to play a crucial role in tumour development, invasion, metastasis and recurrence. Treatments for lung cancer remain a challenging due to ineffectiveness of current available therapies to eradicate the quiescent and therapy-resistance of CSCs. Therefore, new treatment regime specifically targeting CSCs are needed to improve the effectiveness of lung cancer treatment. However the identification of lung CSCs especially in nonsmall cell lung cancer (NSCLC) remains challenging due to highly heterogeneous cell population and no standard markers to distinguish the lung CSCs from their cancer origins. The use of cell surface markers in isolation, identification and characterization of CSCs is one of the important tools as to understand the basic molecular composition and properties of CSCs and the pathways that of these heterogeneous population of cells are involved for future targeted therapy. Our group has identified, isolated and characterized putative lung CSCS from NSCLC cell line based on expression of combination CSCs markers, the CD166/CD44 and CD166/EpCAM using fluorescence activated cell sorting (FACS). The putative lung CSCs, the CD166+CD44+ CD166+EpCAM+ were characterized for multipotent and self-renewal capability using differentiation, clonogenic and spheroid assays. Following microarray gene expression study and bioinformatics analysis, our data has shown that the NF-KB was consistently expressed and shown to play a significant role in maintaining tumorigenicity of CSCs. In order to proof the role of NF-KB, the cells were treated with different concentrations of BMS-345541 (an inhibitor of NF-KB molecule) and the effectiveness of the treatments were evaluated through its ability to reduce self-renewal, expression of stemness genes (Sox2, Oct4, Klf4, Nanog and Sca-1), migration and epithelial to mesenchymal transition process (EMT) activities. The effectiveness of curcumin as drug sensitizer for effective therapy in treating chemoresistance of CSCs, was also investigated. The capability of CD166+/CD44+ and CD166+/EpCAM+ to differentiate into multipotent cell lineages (adipocytes and osteocyte), to form colony and to anchorage independent spheres and expressed stemness genes were shown in this study. The inhibition of NF-KB molecule using BMS-345441 has reduced the sphere forming capability, expression of stemness genes (Sox2, Nanog and Oct4), migration and expression of EMT genes in lung CSCs. Meanwhile, curcumin was found to reduce the cells proliferation activity and induced apoptosis of the cells. In addition, curcumin was also able to sensitize cisplatin, to supress the migration ability and inhibiting self-renewal capability of lung CSCs. Besides, the mRNA level of Sox2, KIf4, Nanog and Oct4 were down-regulated in lung CSCs after treatment with combination of both curcumin and cisplatin. Our findings suggest that NF$\mathrm{KB}$ could be used as a potential targeted gene for lung cancer gene modification whereas curcumin could be used as potential natural compound that was able to sensitize the chemotherapy drug for effective cancer therapy. 
Keywords

cancer stem cells, cell surface markers, microarray, curcumin

Funding

References 